\title{
Multi-component symmetry-projected approach for molecular ground state correlations
}

\author{
Carlos A. Jiménez-Hoyos, ${ }^{1}$ R. Rodríguez-Guzmán, ${ }^{1,2}$ and Gustavo E. Scuseria ${ }^{1,2}$ \\ ${ }^{1}$ Department of Chemistry, Rice University, Houston, Texas 77005, USA \\ ${ }^{2}$ Department of Physics and Astronomy, Rice University, Houston, Texas 77005, USA
}

(Received 17 September 2013; accepted 7 November 2013; published online 22 November 2013)

\begin{abstract}
The symmetry-projected Hartree-Fock ansatz for the electronic structure problem can efficiently account for static correlation in molecules, yet it is often unable to describe dynamic correlation in a balanced manner. Here, we consider a multi-component, systematically improvable approach, that accounts for all ground state correlations. Our approach is based on linear combinations of symmetry-projected configurations built out of a set of non-orthogonal, variationally optimized determinants. The resulting wavefunction preserves the symmetries of the original Hamiltonian even though it is written as a superposition of deformed (broken-symmetry) determinants. We show how short expansions of this kind can provide a very accurate description of the electronic structure of simple chemical systems such as the nitrogen and the water molecules, along the entire dissociation profile. In addition, we apply this multi-component symmetry-projected approach to provide an accurate interconversion profile among the peroxo and bis $\left(\mu\right.$-oxo) forms of $\left[\mathrm{Cu}_{2} \mathrm{O}_{2}\right]^{2+}$, comparable to other state-of-the-art quantum chemical methods. () 2013 AIP Publishing LLC. [http://dx.doi.org/10.1063/1.4832476]
\end{abstract}

\section{INTRODUCTION}

In recent work, ${ }^{1-5}$ we have explored the merits of the symmetry-projected Hartree-Fock (HF) ansatz for describing the electronic structure of molecular systems. A symmetryprojected ansatz can account for most of the static correlations present in molecular systems while also capturing a fraction of the dynamic correlation. The resulting wavefunction has a highly non-trivial multi-determinantal character, with well defined quantum numbers, and yet it is described by a single set of occupied orbitals. In this way, the connection to the single-particle picture is not completely abandoned. The projected state can be expressed as the resonance among the different broken-symmetry, defect-possessing Slater determinants, in such a way that a state with well-defined symmetries is recovered.

A successful many-body approach to quantum chemistry must be able to predict reaction energies and reaction barriers with (near) chemical accuracy. That is, the method must be able to predict energy differences between reactants, products, and transition states to within a few $\mathrm{kcal} / \mathrm{mol}$, which is a small fraction of the total electronic energies. In order to accomplish this task for general chemical systems, the method must provide a balanced description of static and dynamic correlations for the different chemical species participating in a given process. A symmetry-projected HF approach will generally fail these tests: the method is not size consistent and the amount of correlations captured is, to a given extent, system and symmetry dependent.

In this work, we explore a systematic way to approach the exact many-body wavefunction by taking linear combinations of symmetry-projected configurations. Ideally, the multi-component approaches here considered should account for most of the correlations (both static and dynamic) in chemical systems with just a few symmetry-projected configurations. If the number of such configurations depends weakly on the size of the system, the approach remains mean-field in computational cost.

Our multi-component approach follows the fewdeterminant (FED) treatment described by $\mathrm{Schmid}^{6,7}$ in the nuclear physics community as well as the resonating HF (Res HF) approach originally proposed by Fukutome. ${ }^{8}$ The two constitute extreme strategies of a more general method where linear combinations of symmetry-projected configurations are used, regardless of the approach used to optimize them. We note that a linear combination of restricted HF determinants was used by Koch and Dalgaard ${ }^{9}$ to reach near full configuration-interaction (FCI) accuracy in the electronic energies of $\mathrm{Be}, \mathrm{BH}$, and $\mathrm{H}_{2} \mathrm{O}$. Similarly, a Res $\mathrm{HF}$ approach was used by Tomita, Ten-no, and Tanimura ${ }^{10}$ in approximate symmetry-projected calculations on carbon monoxide. In contrast to these previous efforts, our work is characterized by the simultaneous exploitation of linear combinations of non-orthogonal determinants each one symmetry-projected and variationally optimized. This particular approach has not been explored in previous investigations, especially in connection with non-collinear spin projection and spatial symmetry breaking and restoration. Both FED and Res HF approaches based on symmetry-projected configurations have been successfully applied to describe strongly correlated systems in condensed matter physics like the one and two-dimensional Hubbard model. ${ }^{11-14}$ In general terms, the methods described in this paper fall within the category of non-orthogonal configuration interaction approaches of which there are several examples in quantum chemistry. ${ }^{15,16}$

This work is organized as follows. In Sec. II we provide details of our formalism. In particular, after a brief review of the symmetry-projected HF approach, we describe the 
general multi-component approach and then briefly consider the FED and Res HF strategies to optimize the resulting ansatz. In Sec. III we describe some features of our computational implementation. In Sec. IV, we apply the multi-component formalism to describe the correlation in the dissociation profile of $\mathrm{N}_{2}$ and $\mathrm{H}_{2} \mathrm{O}$. We have also considered the challenging $\left[\mathrm{Cu}_{2} \mathrm{O}_{2}\right]^{2+}$ species with our new approach. Finally, Sec. V is devoted to concluding remarks and work perspectives.

\section{FORMALISM}

In this section, we describe in detail the formalism we use. We consider the symmetry-projected HF ansatz for the ground state of a molecular system in Sec. II A. We describe a CI expansion based on symmetry-projected configurations in Sec. II B, which we actually do not use but let us nicely put in perspective our multi-component approach. Finally, in Sec. II C we describe the multi-component approach, focusing in the FED and Res HF strategies used in its optimization.

\section{A. Symmetry-projected Hartree-Fock}

We start this section by clarifying that we understand a symmetry-projected ansatz as a wavefunction where good quantum numbers are restored from a broken-symmetry state even if "true" projection operators (in the strict mathematical sense) are not used. ${ }^{17}$ The symmetry-projected HF ansatz takes the form ${ }^{18}$

$$
\left|\Psi_{j, m}\right\rangle=\sum_{k} f_{k} \hat{P}_{m k}^{j}|\Phi\rangle
$$

where $|\Phi\rangle$ is a broken-symmetry Slater determinant, $\hat{P}_{m k}^{j}$ is a "projection-like" operator (written for general non-Abelian groups) and $\{f\}$ is a set of linear variational coefficients. Here, $j$ labels the irreducible representation and $m$ is used for the row of the irrep; thus $j$ and $m$ constitute good quantum numbers. For one-dimensional irreps, a single quantum number $j$ is sufficient. Upon action of $\hat{P}_{m k}^{j} \equiv|j, m\rangle\langle j, k|$ on $|\Phi\rangle$, the component with $j, k$ quantum numbers is extracted and written in terms of a wavefunction with quantum numbers $j, m$.

The sum in Eq. (1), looping over the dimension of the irreducible representation $j$, is necessary in order to remove an unphysical dependence ${ }^{19}$ of $\left|\Psi_{j, m}\right\rangle$ on the orientation of $|\Phi\rangle$. Consider, for instance, a hydrogen-atom wavefunction. One can recover a state $\left|\Psi_{1 / 2,1 / 2}\right\rangle$ from a determinant $|\Phi\rangle$ with a spin-up electron by acting with $\hat{P}_{1 / 2,1 / 2}^{1 / 2}$ on it. On the other hand, the same state can only be recovered from a spindown electron determinant by acting with $\hat{P}_{1 / 2,-1 / 2}^{1 / 2}$. In general, a linear combination of the form of Eq. (1) removes the dependence upon the orientation of $|\Phi\rangle$.

The symmetry-projected HF ansatz has a long history in quantum chemistry. Originally proposed by Löwdin in 1955 , it was usually associated with spin projection out of an unrestricted reference determinant. ${ }^{20,21}$ Only recently our research group has shown ${ }^{1}$ how to efficiently carry out the fully variational optimization of symmetry-projected HF configurations, borrowing techniques commonly applied in the nuclear physics community that we have adapted to the molecular electronic structure problem. Our strategy allows us to break and restore all symmetries of the molecular Hamiltonian, including those that are not spontaneously broken, in a fully self-consistent variational approach.

The projection operators we use take the generic form

$$
\hat{P}_{m k}^{j}=\frac{1}{V} \int_{V} d \vartheta w_{m k}^{j}(\vartheta) \hat{R}(\vartheta) .
$$

Here, $\vartheta$ labels the elements of the symmetry group; for discrete groups (such as most point groups), the integration should be understood as a summation. In addition, $V$ is the volume of integration, $w_{m k}^{j}(\vartheta)$ is an integration weight (or the character for one-dimensional irreps) associated with the symmetries of the state to be recovered, and $\hat{R}(\vartheta)$ is a rotation operator. We point the interested reader to Refs. 7 and 17 for more details of the form of the projection operators. The spin projection operator, for example, takes the form

$$
\hat{P}_{m k}^{s}=\frac{2 s+1}{8 \pi^{2}} \int d \Omega D_{m k}^{s *}(\Omega) \hat{R}(\Omega),
$$

where $\Omega=(\alpha, \beta, \gamma)$ stands for the set of Euler angles, $D_{m k}^{s}(\Omega)=\langle s, m|R(\Omega)| s, k\rangle$ is an element of Wigner's Dmatrix, and $\hat{R}(\Omega)=\exp \left(-i \alpha \hat{S}_{z}\right) \exp \left(-i \beta \hat{S}_{y}\right) \exp \left(-i \gamma \hat{S}_{z}\right)$ is the spin-rotation operator. Similarly, a projection into any of the irreps of the $C_{2 v}$ group ( $\left.j=A_{1}, A_{2}, B_{1}, B_{2}\right)$ can be accomplished with

$$
\hat{P}^{j}=\frac{1}{4} \sum\left\{\chi(E) \hat{E}+\chi\left(C_{2}\right) \hat{C}_{2}+\chi\left(\sigma_{x z}\right) \hat{\sigma}_{x z}+\chi\left(\sigma_{y z}\right) \hat{\sigma}_{y z}\right\}
$$

where $\chi(\vartheta)$ are the characters of the selected irrep and $\hat{E}$ (identity), $\hat{C}_{2}\left(180^{\circ}\right.$ rotation), and $\hat{\sigma}_{x z}, \hat{\sigma}_{y z}$ (mirror planes) are the elements of the $C_{2 v}$ group.

Given the form (Eq. (2)) of the projection operator, the symmetry-projected HF wavefunction can be expressed as a superposition of states of the form $\hat{R}(\vartheta)|\Phi\rangle$, that is, all degenerate states (the Goldstone manifold) generated by the set of operators commuting with the Hamiltonian. ${ }^{22}$ The coefficients $w_{m k}^{j}(\vartheta)$ are fully determined by the irrep to be recovered.

The energy of the ansatz of Eq. (1) is given by

$$
\begin{aligned}
E_{j}[\Phi] & =\frac{\sum_{k k^{\prime}} f_{k}^{*} f_{k^{\prime}}\left\langle\Phi\left|\hat{P}_{m k}^{j \dagger} \hat{H} \hat{P}_{m k^{\prime}}^{j}\right| \Phi\right\rangle}{\sum_{k k^{\prime}} f_{k}^{*} f_{k^{\prime}}\left\langle\Phi\left|\hat{P}_{m k}^{j \dagger} \hat{P}_{m k^{\prime}}^{j}\right| \Phi\right\rangle} \\
& =\frac{\sum_{k k^{\prime}} f_{k}^{*} f_{k^{\prime}}\left\langle\Phi\left|\hat{H} \hat{P}_{k k^{\prime}}^{j}\right| \Phi\right\rangle}{\sum_{k k^{\prime}} f_{k}^{*} f_{k^{\prime}}\left\langle\Phi\left|\hat{P}_{k k^{\prime}}^{j}\right| \Phi\right\rangle}=\frac{\sum_{k k^{\prime}} f_{k}^{*} f_{k^{\prime}} \mathcal{H}_{k k^{\prime}}}{\sum_{k k^{\prime}} f_{k}^{*} f_{k^{\prime}} \mathcal{N}_{k k^{\prime}}}
\end{aligned}
$$

Here, we have used the properties of the "projection-like" operators

$$
\begin{aligned}
\hat{P}_{\lambda \kappa}^{j} \hat{P}_{\mu \nu}^{k} & =\delta_{j k} \delta_{\kappa \mu} \hat{P}_{\lambda \nu}^{k}, \\
\left(\hat{P}_{\lambda \kappa}^{j}\right)^{\dagger} & =\hat{P}_{\kappa \lambda}^{j} .
\end{aligned}
$$

Note that the energy only depends on the irrep $j$ recovered, but not on the quantum number $m$ labelling the row of the irrep.

The matrix elements appearing in Eq. (5) can be efficiently evaluated using the formulas provided in the Appendix. The corresponding derivation of the matrix elements 
can be found in, e.g., Ref. 23. For a detailed discussion of how the ansatz of Eq. (1) is optimized with respect to the set of linear variational coefficients $\{f\}$ and with respect to the underlying broken symmetry determinant $|\Phi\rangle$ we refer the reader to Refs. 23, 24. We stress that the optimization method that we follow is different from the one used in Ref. 1 , where a parametrization based on the density matrix of the deformed determinant was used. We note that a stationary point is found when the following equations are all satisfied:

$$
\begin{aligned}
\sum_{k k^{\prime}} f_{k}^{*} f_{k^{\prime}} \mathcal{N}_{k k^{\prime}} & =1, \\
\sum_{k^{\prime}}\left(\mathcal{H}_{k k^{\prime}}-E \mathcal{N}_{k k^{\prime}}\right) f_{k^{\prime}} & =0 \quad \forall \quad k, \\
\frac{\sum_{k k^{\prime}} f_{k}^{*} f_{k^{\prime}}\left\langle\Phi_{i}^{a}\left|(\hat{H}-E) P_{k k^{\prime}}^{j}\right| \Phi\right\rangle}{\sum_{k k^{\prime}} f_{k}^{*} f_{k^{\prime}} \mathcal{N}_{k k^{\prime}}} & =0 \quad \forall \quad i, a .
\end{aligned}
$$

Here, $E$ is the energy of Eq. (5) and $\left|\Phi_{i}^{a}\right\rangle$ is a singly excited determinant $(i \rightarrow a)$ built from $|\Phi\rangle$. The second equation determines a generalized eigenvalue problem for the coefficients $\{f\}$ subject to the normalization constraint expressed by the first equation. Only the lowest energy eigenvector of the eigenvalue problem is considered. The last equation constitutes the generalized Brillouin condition decoupling the ground-state solution from excited particle-hole configurations.

\section{B. Configuration interaction based on symmetry-projected determinants}

Conceptually, the simplest approach to account for missing correlations in the symmetry-projected HF ansatz is to consider a configuration interaction approach. A configuration interaction ansatz can be written as

$$
\begin{aligned}
\left|\Psi_{j, m}\right\rangle= & \sum_{k} \hat{P}_{m k}^{j}\left(f_{0 ; k}|\Phi\rangle+\sum_{i a, k} f_{i a ; k}\left|\Phi_{i}^{a}\right\rangle\right. \\
& \left.+\sum_{i j a b, k} f_{i a, j b ; k}\left|\Phi_{i j}^{a b}\right\rangle+\cdots\right) .
\end{aligned}
$$

In the above expression, we have used indices $i$ and $j$ to denote occupied (hole) states in the broken-symmetry determinant $|\Phi\rangle$, whereas indices $a$ and $b$ are used for unoccupied (particle) states. The state $\left|\Phi_{i}^{a}\right\rangle$ constitutes a singly excited determinant out of the reference Fermi vacuum $|\Phi\rangle$. The linear variational coefficients $f$ can be determined by the solution to a generalized eigenvalue problem among all configurations. We note that the above representation of the Hilbert subspace with the appropriate symmetry is overcomplete.

Including only singly excited configurations (of the form $\left.\left|\Phi_{i}^{a}\right\rangle\right)$ in the configuration-interaction expansion will in general not lead to any improvement in the ground state energy. In particular, the generalized Brillouin condition that a variationally optimized symmetry-projected HF state satisfies is given by Eq. (9), which makes singly excited configurations orthogonal to the symmetry-projected HF state through the Hamiltonian when using the $f_{0 ; k}$ variational coefficients. Note that if the dimension of the irreducible representation associated with the restored symmetry is larger than 1, some energy improvement in the ground state may be obtained by diagonalization in the singly excited space due to the variational coefficients $f_{i a, k}$. On the other hand, diagonalization in this space can be used for a first-order description of excited states. This corresponds to the symmetry-projected Tamm-Dancoff approximation discussed by Schmid et al. ${ }^{6}$

If, in addition, one includes doubly excited configurations, an energy improvement is all but guaranteed unless the symmetry-projected HF state was already exact. Nevertheless, the matrix is large and dense; the evaluation of each matrix element is significantly more expensive than in the standard HF-based approach, where the Slater-Condon rules ${ }^{25}$ can be used to simplify the evaluation.

Given that the representation of the Hamiltonian is not sparse among symmetry-projected configurations, even if built from orthogonal, particle-hole excited determinants, the above CI-like approach cannot be considered efficient. Note, for instance, that including all doubly excited configurations yields an $\mathcal{O}\left(M^{6}\right)$ eigenvalue problem if carried out straightforwardly. The construction of the matrix is even more expensive as each matrix element can only be evaluated at mean-field $\left(\mathcal{O}\left(M^{4}\right)\right)$ cost.

We have thus explored an alternative approach: we consider expansions based on a few symmetry-projected configurations resulting from a set of non-orthogonal determinants. The use of non-orthogonal determinants allows us to arrive at a much more compact representation than would be possible using an expansion in terms of orthogonal determinants while accessing even highly excited configurations. A non-orthogonal expansion also facilitates the physical insight behind the wavefunction: the correlation thus gained can be attributed to the resonance among the configurations in the expansion. If the number of configurations needed in the expansion is essentially constant with increasing system size, the method has mean-field computational cost. We note that this spirit is also pursued in the generalized multistructural wavefunction of Hollauer and Nascimento, ${ }^{16}$ where a linear combination of expansions based on non-orthogonal Slater determinants is used as a variational ansatz.

\section{Multi-component approaches}

Let us suppose that we have already optimized a symmetry-projected HF configuration. In this section, we write this as

$$
\left|{ }^{1} \Psi_{j, m}\right\rangle=\sum_{k} f_{k}^{1} \hat{P}_{m k}^{j}\left|{ }^{1} \Phi\right\rangle,
$$

where the superscript 1 in $\left|{ }^{1} \Psi_{j, m}\right\rangle$ is used to indicate that a single symmetry-projected configuration is used in the ansatz. Similarly, the superscript 1 in the $f$ variational coefficients and in $|\Phi\rangle$ indicates that only one determinant is included in the ansatz.

In the general case, we can describe the ground state with $n$ symmetry-projected configurations, as in

$$
\left.\left|{ }^{n} \Psi_{j, m}\right\rangle=\left.\sum_{k} \hat{P}_{m k}^{j} \sum_{l=1}^{n} f_{k}^{l}\right|^{l} \Phi\right\rangle .
$$


Note that this defines a systematically improvable approach. That is, if $n$ determinants prove insufficient to provide an accurate description of a given system, one can add one (or a few) more configuration(s) to the expansion. It is also important to note that the expansion of Eq. (12) is written as a superposition of the Goldstone manifolds associated with each of the $n$-deformed determinants in the multi-component wavefunction. One has now to address the issue of how to variationally optimize the $n$-configuration ansatz. There are two extreme approaches that we will consider:

- In the FED approach, $, 6,11$ the different configurations are optimized one-at-a-time. That is, the second symmetry-projected configuration is optimized after the first one, leaving the latter untouched, and so on.

We note that there is no need for the FED expansion to be short, as its name would imply, although it is a desirable feature. We keep the acronym to remain consistent with the literature.

- In the resonating HF (Res HF) approach, ${ }^{8}$ all the configurations are optimized at the same time.

There are, of course, a number of possible variants in between. For instance, one could optimize two configurations at a time. Each approach has strengths and drawbacks. In particular, we would like to stress the following:

- A Res HF optimized wavefunction is stationary with respect to changes in any of the underlying determinants. On the other hand, a FED optimized wavefunction is stationary only with respect to particle-hole excitations of the last-added determinant.

This feature makes the Res HF wavefunction easier to work with for evaluating properties that depend on derivatives of the wavefunction.

- In a Res HF optimization, $\mathcal{O}\left(n^{2}\right)$ overlap and Hamiltonian matrix elements need to be re-computed at every iteration. In contrast, an efficient implementation of the FED approach requires only $\mathcal{O}(n)$ overlap and Hamiltonian matrix elements to be recomputed.

- The convergence properties of the two approaches can be very different. In the Res HF approach, for instance, there is no guarantee that any of the configurations will resemble the optimized single-configuration ansatz.

The matrix elements appearing in the evaluation of the energy and energy gradient with multi-component approaches can be efficiently evaluated using the expressions provided in the Appendix. In our calculations, we carry out the optimization with respect to the broken-symmetry determinants in the multi-component expansion using a robust, Thoulessbased parametrization. ${ }^{26,27}$ We now proceed to consider each of the two approaches in detail.

\section{The few-determinant approach}

In the FED approach introduced by Schmid, ${ }^{6,7}$ only the last-added symmetry-projected configuration is optimized with respect to the underlying $\mathrm{HF}$ transformation. In the quantum chemistry community, a similar approach was proposed by Koch and Dalgaard, ${ }^{9}$ although the configurations included were limited to a restricted HF-type ansatz. The FED approach has been very successful in the nuclear physics community (see Ref. 7 and references therein).

Let us consider the variational optimization of the $n$th determinant in the ansatz defined by Eq. (12). The energy functional becomes

$$
\begin{aligned}
{ }^{n} E_{j}\left[{ }^{n} \Phi,\{f\}\right] & =\frac{\left\langle{ }^{n} \Psi_{j, m}|\hat{H}|^{n} \Psi_{j, m}\right\rangle}{\left\langle{ }^{n} \Psi_{j, m} \mid{ }^{n} \Psi_{j, m}\right\rangle} \\
& =\frac{\sum_{k l, k^{\prime} l^{\prime}} f_{k}^{l *} f_{k^{\prime}}^{l^{\prime}}\left|{ }^{l} \Phi\right| \hat{H} \hat{P}_{k k^{\prime}}^{j}\left|l^{l^{\prime}} \Phi\right\rangle}{\sum_{k l, k^{\prime} l^{\prime}} f_{k}^{l *} f_{k^{\prime}}^{l^{\prime}}|l| \hat{P}_{k k^{\prime}}^{j}\left|l^{\prime} \Phi\right\rangle} .
\end{aligned}
$$

Here, we use the symbol ${ }^{n} E_{j}$ for the energy of the state to denote that it corresponds to an $n$-determinant expansion and to emphasize that it only depends on the label $j$ of the irreducible representation but not on the row $m$ projected.

The variation with respect to the coefficients $f$ (note that the full set is re-optimized) leads to the generalized eigenvalue problem

$$
\sum_{k^{\prime} l^{\prime}}\left({ }^{n} \mathcal{H}_{k l, k^{\prime} l^{\prime}}-{ }^{n} E^{n} \mathcal{N}_{k l, k^{\prime} l^{\prime}}\right) f_{k^{\prime}}^{l^{\prime}}=0 \quad \forall \quad k, l,
$$

subject to the constraint

$$
\sum_{k l, k^{\prime} l^{\prime}} f_{k}^{l * n} \mathcal{N}_{k l, k^{\prime} l^{\prime}} f_{k^{\prime}}^{l^{\prime}}=1
$$

Here, the matrices ${ }^{n} \mathcal{H}$ and ${ }^{n} \mathcal{N}$ are given by

$$
\begin{gathered}
{ }^{n} \mathcal{H}_{k l, k^{\prime} l^{\prime}}=\left\langle{ }^{l} \Phi\left|\hat{H} \hat{P}_{k k^{\prime}}^{j}\right| l^{l^{\prime}} \Phi\right\rangle, \\
{ }^{n} \mathcal{N}_{k l, k^{\prime} l^{\prime}}=\left\langle{ }^{l} \Phi\left|\hat{P}_{k k^{\prime}}^{j}\right|{ }^{l^{\prime}} \Phi\right\rangle .
\end{gathered}
$$

A stationary point in the optimization with respect to $\left|{ }^{n} \Phi\right\rangle$ is found when

$$
\frac{\sum_{k, k^{\prime} l^{\prime}} f_{k}^{n *} f_{k^{\prime}}^{l^{\prime}}\left|{ }^{n} \Phi_{i}^{a}\right|\left(\hat{H}-{ }^{n} E\right) \hat{P}_{k k^{\prime}}^{j}\left|l^{\prime} \Phi\right\rangle}{\left.\left.\sum_{k l, k^{\prime} l^{\prime}} f_{k}^{l *} f_{k^{\prime}}^{l^{\prime}}|l \Phi| \hat{P}_{k k^{\prime}}^{j}\right|^{l^{\prime}} \Phi\right\rangle}=0 \quad \forall \quad i, a .
$$

Because the states are constructed using a chain of variational calculations, one can easily prove that

$$
{ }^{1} E-{ }^{2} E \geq{ }^{2} E-{ }^{3} E \geq \cdots \geq{ }^{n-1} E-{ }^{n} E .
$$

That is, the last added symmetry-projected configuration will improve the ground state energy by a smaller amount than the previously added one. Of course this is only satisfied if one can guarantee that the global minimum was found in each optimization problem. In practice, as this is difficult to guarantee, small deviations to this rule are observed, yet the overall trend remains valid.

We close this section by noting that Schmid et al. ${ }^{6}$ realized that the FED approach is not the most general description using $n$ symmetry-projected configurations. The authors stated, regarding the Res HF approach discussed in Sec. II C 2, that they did not believe that "such a fine-tuning will yield improvements with respect to the (FED) approach." 


\section{The resonating HF approach}

It is perhaps conceptually simpler, though computationally more challenging, to optimize all configurations at the same time. This is the basis of the resonating Hartree-Fock method devised by Fukutome. ${ }^{8}$ It has been used by Tomita, Ten-no, and Tanimura ${ }^{10}$ in approximate symmetry-projected Res HF calculations on CO (carbon monoxide), and by Tenno in CI and coupled-cluster approaches based on a Res HF expansion. ${ }^{28}$ It has proven very successful in the context of the Hubbard model, ${ }^{12-14,29}$ which can be regarded as a oneorbital-per-site cluster Hamiltonian.

Let us consider the variational optimization of the ansatz defined by Eq. (12). The energy functional becomes

$$
\begin{aligned}
{ }^{n} E_{j}[\{\Phi\},\{f\}] & =\frac{\left\langle{ }^{n} \Psi_{j, m}|\hat{H}|^{n} \Psi_{j, m}\right\rangle}{\left\langle{ }^{n} \Psi_{j, m} \mid{ }^{n} \Psi_{j, m}\right\rangle} \\
& =\frac{\sum_{k l, k^{\prime} l^{\prime}} f_{k}^{l *} f_{k^{\prime}}^{l^{\prime}}\left|{ }^{l} \Phi\right| \hat{H} \hat{P}_{k k^{\prime}}^{j}\left|l^{l^{\prime}} \Phi\right\rangle}{\sum_{k l, k^{\prime} l^{\prime}} f_{k}^{l *} f_{k^{\prime}}^{l^{\prime}}\left|{ }^{l} \Phi\right| \hat{P}_{k k^{\prime}}^{j}\left|l^{\prime} \Phi\right\rangle},
\end{aligned}
$$

where we have emphasized that the full set of determinants is optimized. Note that the form of the energy expression is the same as in the FED approach; the difference lies in the variational flexibility. The variation with respect to the coefficients $f$ leads to the same generalized eigenvalue problem as in the FED approach (though the matrix elements are necessarily different).

A stationary point in the optimization with respect to $\{\Phi\}$ is achieved when

$$
\frac{\left.\left.\sum_{k l, k^{\prime} l^{\prime}} f_{k}^{l *} f_{k^{\prime}}^{l^{\prime}}\right|^{l} \Phi_{i}^{a}\left|\left(\hat{H}-{ }^{n} E\right) \hat{P}_{k k^{\prime}}^{j}\right|{ }^{l^{\prime}} \Phi\right\rangle}{\left.\sum_{k l^{\prime \prime}, k^{\prime} l^{\prime}} f_{k}^{l^{\prime \prime} *} f_{k^{\prime}}^{l^{\prime}} l^{l^{\prime \prime}} \Phi\left|\hat{P}_{k k^{\prime}}^{j}\right|^{l^{\prime}} \Phi\right\rangle}=0 \quad \forall \quad l, i, a .
$$

This implies that the Res HF wavefunction is stationary with respect to particle-hole mixings of any of the determinants in the expansion.

\section{COMPUTATIONAL DETAILS}

We have implemented the multi-component symmetryprojected HF approach for molecular systems in an in-house program. One- and two-electron integrals are extracted from the Gaussian $09^{30}$ suite. Our program is parallelized (a hybrid openMP/MPI approach is used) over the grid-points used in the symmetry-projection as well as over the configurations used in the multi-component expansion. Our program is currently limited to the use of Cartesian gaussian basis sets. We note that our FED-type implementation re-uses overlap and Hamiltonian matrix elements and thus scales as $\mathcal{O}(n)$ with the number of symmetry-projected configurations. ${ }^{11}$

The optimization of the broken-symmetry determinants is carried out using a Thouless-based strategy, as described in detail in Refs. 24, 27 and 31, with a limited-memory quasi-Newton approach. ${ }^{32,33}$

One of the most important issues regarding a practical implementation of the FED and Res HF approaches is to prepare an initial guess of the underlying HF determinants in the symmetry-projected configuration expansion. This was discussed in some detail by Koch and Dalgaard. ${ }^{9}$ Our approach is currently simplistic: we prepare an initial guess of the HF determinants in the FED approach as random unitary rotations of the orbitals closest to the Fermi energy in the standard HF determinant or the optimized determinant in a one-configuration symmetry-projected expansion. The unitary matrix is built in the form $\exp (i \lambda K)$, with $\lambda \approx 0.01$ and $K$ being a Hermitian matrix. Our initial guess for Res HF calculations is the converged FED expansion with the same number of determinants. Given that the symmetry-projected FED or Res HF equations will reach a stationary point depending on the initial guess provided, a smarter scheme to prepare the initial guess is desirable. Nevertheless, it is difficult to anticipate a priori the structure of general non-orthogonal determinants that will interact the most through the Hamiltonian with the set of previously obtained determinants. Finally, we would like to point out that in order to obtain continuous dissociation profiles in the examples provided, we have used the determinant obtained in a neighboring point in the potential energy curve as an initial guess for the solution.

Before we discuss our results, let us briefly clarify the nomenclature we use. All symmetry projected methods are written in the form X-Y. Here, $\mathrm{Y}=\mathrm{RHF}$ (restricted), UHF (unrestricted), or GHF (generalized) denotes the type of underlying HF determinant used; complex orbitals are used in all our multi-component calculations. In X, we write the collection of symmetries restored in the calculation: $\mathrm{S}$ is used for spin and the point group label (like $C_{2 v}$ ) is used to denote the type of spatial symmetry projection.

\section{RESULTS AND DISCUSSION}

\section{A. Ground-state energy of $\mathrm{N}_{2}$}

We start by considering the ground state energy of the nitrogen molecule, both at the equilibrium geometry $\left(r_{e q}\right)$ and at the recoupling region $\left(1.5 r_{\text {eq }}\right)$, where $r_{\text {eq }}=1.09768 \AA{ }^{34}$ We show in Fig. 1 the evolution of the energy with the number of configurations $(n)$ added in a FED-type expansion for a series of (symmetry-projected) methods. Calculations were performed using the Cartesian cc-pVDZ basis set. We compare our results with coupled-cluster singles and doubles (CCSD) [full] and $\operatorname{CCSD}(\mathrm{T})$ [full] reference energies (obtained with the Gaussian 09 suite). For spin projected methods, projection to the singlet state was carried out; for methods involving spatial symmetry projection, projection was done to the totally symmetric irreducible representation.

A few details of the RHF, UHF, and CCSD calculations in Fig. 1 are in order. At $r_{\text {eq }}$, RHF converges to a symmetry adapted state, which was also used as reference for the coupled-cluster calculations. The energies are -108.9547 , -109.2740 , and -109.2863 hartree, respectively, at the RHF, CCSD [full], and CCSD(T) [full] levels. At $1.5 r_{\text {eq }}$, we have used the lowest energy broken symmetry complex RHF and UHF states; their energies are -108.6336 and -108.7809 hartree, respectively. The corresponding UCCSD [full] and UCCSD(T) [full] energies, which use the UHF state as a reference, are -109.0367 and -109.0507 hartree. 


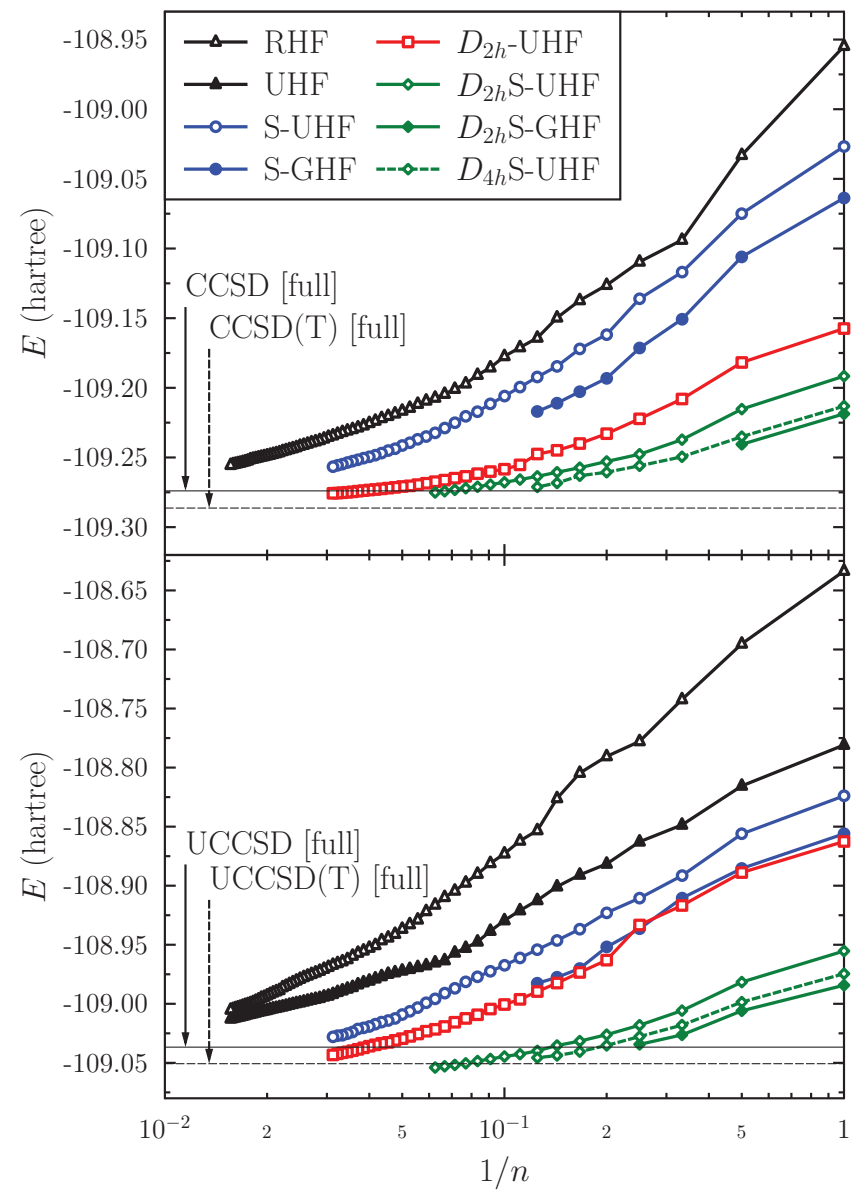

FIG. 1. Ground-state energy of the $\mathrm{N}_{2}$ molecule predicted by a variety of FED approaches at $r=r_{\mathrm{eq}}$ (top panel) and $r=1.5 r_{\mathrm{eq}}$ (bottom panel) as a function of the number $n$ of symmetry-projected configurations. Here, $r_{\mathrm{eq}}$ $=1.09768 \AA ;{ }^{34}$ a Cartesian cc-pVDZ basis set is used. The straight lines in each panel mark the CCSD [full] and CCSD(T) [full] reference energies.

We observe from the results in Fig. 1 that the rule stating that the last added determinant (in FED-type expansions) should bring less correlation than the previously added one is satisfied in most cases. In those cases where it is not, this is because we have failed to converge to the global minimum in the parameter hypersurface. Several other features deserve further discussion:

- At equilibrium, spatial symmetry projection (with the $D_{2 h}$ group) brings significantly more correlation than spin projection with the same number of symmetryprojected configurations. This is not too obvious at $1.5 r_{\text {eq }}$, yet $D_{2 h}$-UHF remains competitive with S-GHF while being significantly cheaper.

- The use of broken spin-symmetry determinants (UHFtype) brings significantly more correlation than the use of RHF determinants at $1.5 r_{\text {eq }}$. This remains true even when several configurations have been added; it takes roughly 4 RHF configurations to obtain the same energy as a single UHF configuration.

- When both spin and spatial symmetry are restored, a small number of configurations seem to be sufficient to obtain energies of comparable quality to CCSD or $\operatorname{CCSD}(\mathrm{T})$. At $1.5 r_{\mathrm{eq}}, \approx 16 D_{2 h} \mathrm{~S}$-UHF configurations
TABLE I. Ground-state energy of the nitrogen molecule (at $r=r_{\mathrm{eq}}$ ) predicted with the multi-component S-UHF approaches as a function of the number of configurations $n$. The Cartesian cc-pVDZ basis set was used.

\begin{tabular}{llr}
\hline \hline$n$ & FED S-UHF & Res HF S-UHF \\
\hline 1 & -109.0267 & -109.0267 \\
2 & -109.0749 & -109.1210 \\
3 & -109.1170 & -109.1530 \\
4 & -109.1360 & -109.1728 \\
5 & -109.1617 & \\
6 & -109.1720 & \\
7 & -109.1845 & \\
8 & -109.1922 & \\
\hline \hline
\end{tabular}

bring more correlation than $\operatorname{CCSD}(\mathrm{T})$. This is remarkable considering the ease of interpretation associated with the multi-component wavefunction.

Unfortunately, we were unable to produce such a detailed plot using the Res HF approach, as it becomes significantly more difficult to converge than the corresponding FED expansion. We show, nonetheless, in Table I a comparison of ground-state energies, evaluated at $r_{\text {eq }}$, predicted with FED S-UHF and Res HF S-UHF as a function of the number of configurations $n$.

It is evident from the results in Table I that the Res HF approach yields significantly lower energies than the FED approach for a fixed number of configurations. However, the FED approach allows one to include many more configurations than in the Res HF approach as the optimization is cheaper and typically takes much fewer iterations with our gradient-based optimization. For instance, Fig. 1 includes results with up to 32 FED SUHF configurations. This makes the FED approach much more convenient for practical applications.

\section{B. Dissociation profiles}

Let us now consider the full dissociation profile of the $\mathrm{N}_{2}$ molecule. Dissociation curves predicted with a FED $D_{2 h} \mathrm{~S}$ UHF approach are shown in Fig. 2, along with the dissociation profile computed with a single symmetry-projected configuration using $D_{2 h} \mathrm{~S}-\mathrm{GHF}$. The calculations use the Cartesian ccpVDZ basis set. We compare our curves with the FCI profile from Ref. 35. Nevertheless, we stress that the FCI results are not directly comparable: they were obtained with the spherical cc-pVDZ basis and freezing the $1 s$ core orbital of the $\mathrm{N}$ atoms. Both of these effects would contribute to underestimate the FCI energy. ${ }^{36}$ The FCI results are included as a guide to the eye for the correct shape of the dissociation curve.

The results in Fig. 2 show that $D_{2 h} \mathrm{~S}$-UHF yields a qualitatively correct dissociation curve even with a single symmetry-projected configuration. Inclusion of 8 symmetryprojected configurations (using the FED approach) results in a curve fairly parallel to the reference FCI curve. The energy improvement due to the additional configurations is seen across the potential energy surface. Interestingly, 2 symmetryprojected configurations with the $D_{2 h} \mathrm{~S}-\mathrm{UHF}$ method match the results from $D_{2 h} \mathrm{~S}-\mathrm{GHF}$ with a single configuration. 


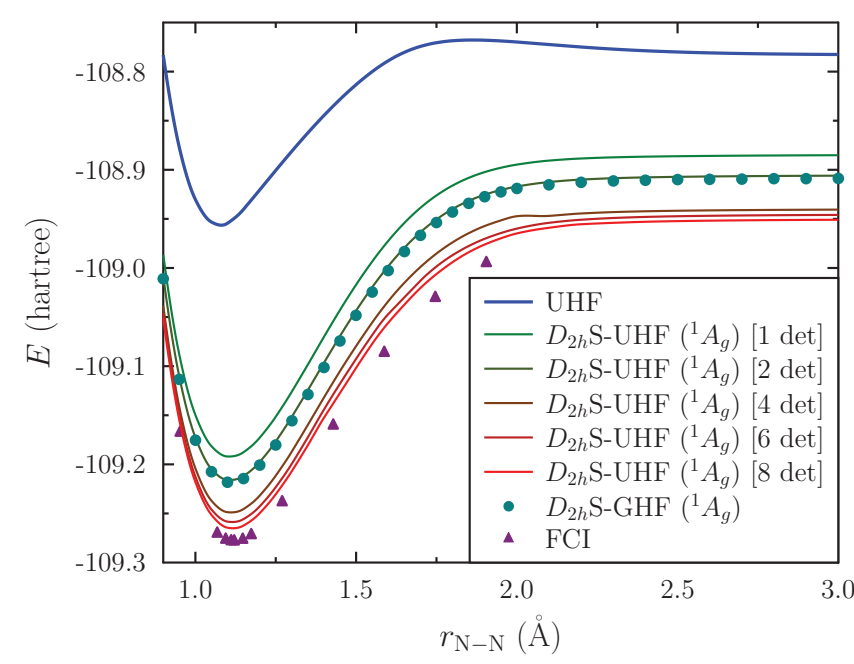

FIG. 2. Dissociation profile for the ground state of the $\mathrm{N}_{2}$ molecule obtained with a FED $D_{2 h}$ S-UHF approach as a function of the number of HF configurations. The single-configuration $D_{2 h} \mathrm{~S}$-GHF profile is included for comparison, as well as the FCI results from Ref. 35. The FCI results use spherical basis functions and frozen $1 s$ orbitals (see text).

Finally, let us stress that the wavefunction resulting from a multi-component approach can be regarded as a discretized form of the unitary group coherent state representation of the exact many-fermion state. ${ }^{37}$ Therefore, in the limit of a large number of configurations, the size-consistency error associated with projected HF approaches necessarily disappears.

We consider in Fig. 3 the symmetric dissociation profile of the $\mathrm{H}_{2} \mathrm{O}$ molecule, as predicted with a variety of multiconfiguration symmetry-projected approaches. The Cartesian cc-pVDZ basis set was used in our calculations, whereas the FCI results from Ref. 38 were obtained with the spherical cc-pVDZ basis set. ${ }^{39}$ The restored quantum numbers in symmetry-projected calculations are $s=0$ for spin and the $A_{1}$ irreducible representation of the $C_{2 v}$ group.

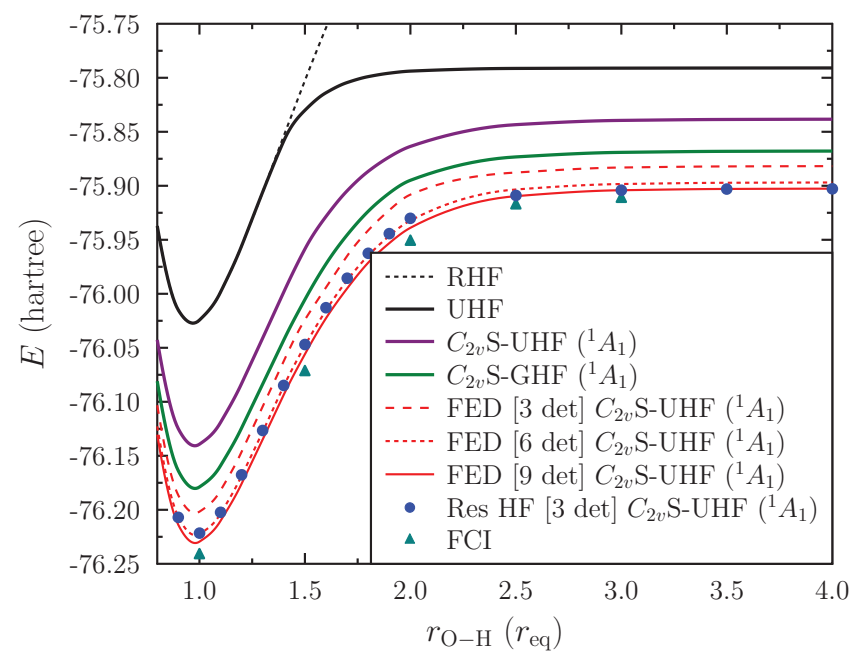

FIG. 3. Symmetric dissociation profile of the water molecule as predicted with a variety of multi-configuration symmetry-projected approaches. A Cartesian cc-pVDZ basis set was used. FCI results from Ref. 38. The FCI results use spherical basis functions (see text).
Results from Fig. 3 show a similar scenario as that observed in the $\mathrm{N}_{2}$ dissociation. The $C_{2 v} \mathrm{~S}$-UHF dissociation profile is already fairly parallel to the FCI solution. It accounts for significantly more correlations near equilibrium than towards dissociation with respect to the UHF solution. With 9 symmetry-projected configurations, the FED $C_{2 v} \mathrm{~S}$ UHF curve is only a few mhartree off from the FCI curve across the entire potential energy surface.

We have been able to compute the entire dissociation profile using a 3-configuration Res HF approach based on $C_{2 v} \mathrm{~S}$-UHF. Interestingly, it yields similar results as a 6-configuration FED approach near equilibrium, but becomes more accurate towards dissociation, rivaling the 9configuration FED approach. This is a result of the increased flexibility in the Res HF ansatz. Finally, Fig. 3 also shows the dissociation profile predicted with the $C_{2 v}$ S-GHF method, using a single configuration. Quite disappointingly, the results are only comparable to a two-configuration $C_{2 v} \mathrm{~S}-\mathrm{UHF}$ wavefunction, even though the former is almost two orders of magnitude more expensive to evaluate.

\section{The copper oxide $\left[\mathrm{Cu}_{2} \mathrm{O}_{2}\right]^{2+}$ core}

We have recently applied the projected Hartree-Fock method $^{2}$ to the theoretical study of the copper oxide cores, in particular, the interconversion profile between the $\mu-\eta^{2}: \eta^{2}$ peroxodicopper(II) (A) and the bis( $\mu$-oxo)-dicopper(II) (B) forms (Fig. 4).

The interconversion profile of the bare $\left[\mathrm{Cu}_{2} \mathrm{O}_{2}\right]^{2+}$ core has been recently studied theoretically by Cramer et al. ${ }^{40}$ Malmqvist et al. ${ }^{41}$ and Yanai et al. ${ }^{42}$ with a variety of highly sophisticated $a b$ initio methods. This system has proven tremendously challenging due to the expected multi-reference character in $\mathbf{A}$ and the large active space that one has to include in traditional multi-reference approaches (a reasonable active space for this system would involve 30 electrons in 28 orbitals).

It should be pointed out that recently Liakos and Neese ${ }^{43}$ have shown that the multi-reference character in the copper oxide core is very limited. They examined the influence of ligands as well as relativistic and solvent effects and concluded that the single-reference based local-pair natural orbital coupled-cluster method in fact provides very reliable profiles for this system. Their assessment is likely valid in the presence of ligands and solvent, and is hence relevant for comparison with experimental results. On the other hand, we can still treat the bare copper oxide core as a toy system for which different highly sophisticated theoretical methods yield inconsistent results.

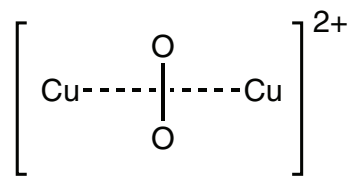

(A)

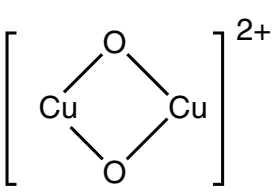

(B)
FIG. 4. Structures of $\mu-\eta^{2}: \eta^{2}$-peroxodicopper(II) (A) and bis( $\mu$-oxo)dicopper(II) (B) in the interconversion profile of the $\left[\mathrm{Cu}_{2} \mathrm{O}_{2}\right]^{2+}$ core. 
In Ref. 2 we assessed the ability of single-reference symmetry-projected methods to accurately describe the interconversion profile of the bare copper oxide core. Understanding that the RASPT2 (restricted active-space second order perturbation theory), ${ }^{41}$ CR-CC (completely renormalized coupled-cluster), ${ }^{40}$ and DMRG-SC-CTSD (density-matrix renormalization group with strongly contracted canonical transformation including only single and double excitations $)^{42}$ methods provide the likely correct profile for this system, we ranked the S-UHF, S-GHF, KS-UHF, and KS-GHF methods according to how close they came to the former methods. We observed that the more symmetries restored the closer the profile got to the reference methods. We show, in the lower panel of Fig. 5, a summary of the results presented in Ref. 2.

We have revisited our results for the interconversion profile of the copper oxide core with our multi-reference FED approaches. We have employed the same basis set as our previous work, save for the fact that our program cannot currently handle spherical basis sets. The effect of the change of basis is expected to be very small and should not affect the

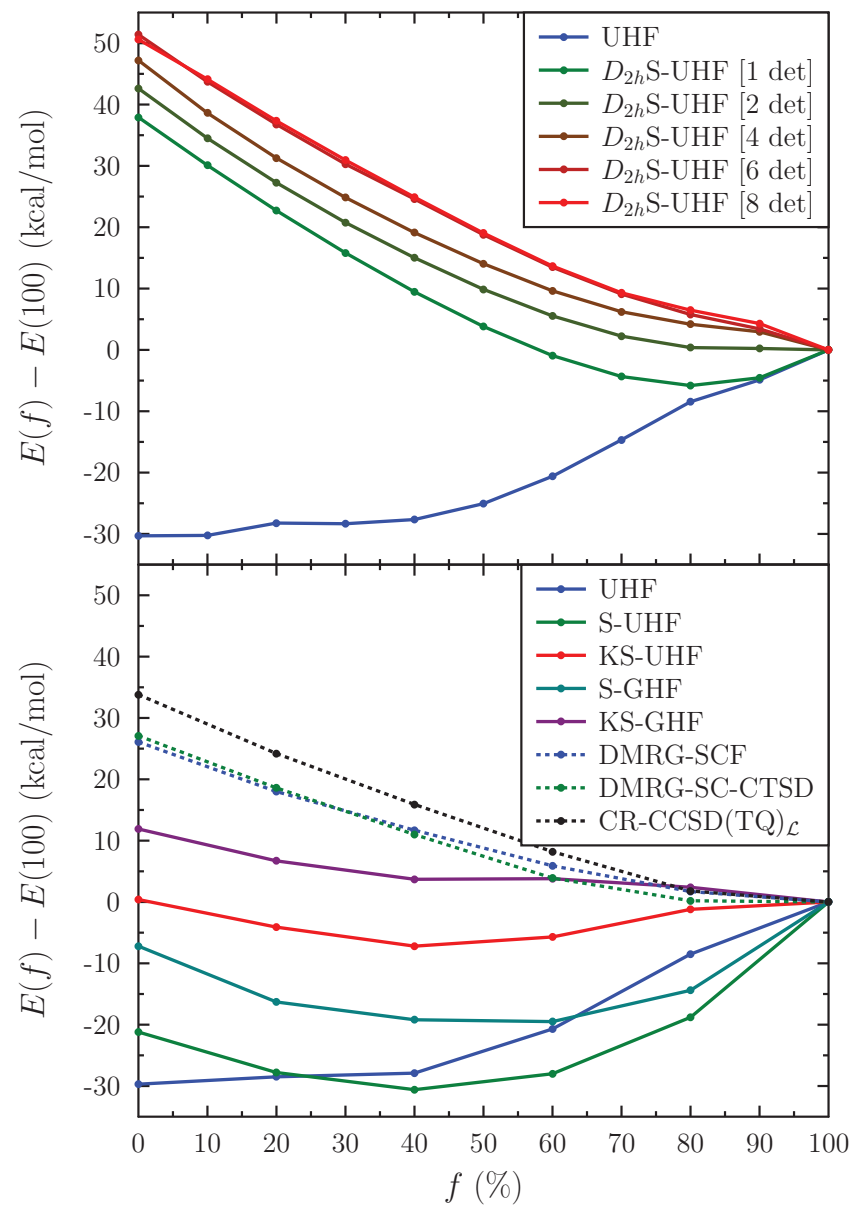

FIG. 5. Relative total energy of $\left[\mathrm{Cu}_{2} \mathrm{O}_{2}\right]^{2+}$ along a linear isomerization path from $\mathbf{B}(f=0)$ to $\mathbf{A}(f=100)$. A variety of single symmetry-projected configuration methods are shown in the bottom panel, while a multi-reference FED approach based on $D_{2 h} \mathrm{~S}$-UHF is shown in the top panel. CR-CC data were extracted from Ref. 40, while DMRG data were taken from Ref. 42. The upper panel uses a Cartesian basis set while the lower panel uses spherical basis functions (see text). conclusions of our work. ${ }^{44}$ The totally symmetric irrep of the $D_{2 h}$ group was restored in our calculations.

The upper panel of Fig. 5 shows the interconversion profiles obtained by our FED $D_{2 h}$ S-UHF approach as a function of the number of configurations included. We note that the restoration of spatial symmetry makes a huge difference even when a single configuration is included. A single-determinant $D_{2 h} \mathrm{~S}$-UHF approach predicts $\mathrm{A}$ to be $\approx 38 \mathrm{kcal} / \mathrm{mol}$ higher in energy than $\mathbf{B}$, and the profile closely resembles those that we have deemed as accurate. Increasing the number of determinants further raises the energy of $\mathbf{A}$ relative to $\mathbf{B}$. Our interconversion profile seems to converge with 6 symmetry-projected configurations to a relative energy of $\approx 50 \mathrm{kcal} / \mathrm{mol}$, notably higher than the RASPT2, CR-CC, and DMRG-SC-CTSD curves. Because our results show a relatively smooth convergence with the number of configurations, we believe our results could be more accurate than the ones just quoted.

\section{CONCLUSIONS}

In this work we have considered a multi-component approach to account for the correlations missing in the symmetry-projected ansatz for the ground state of a molecular system. The ground state description is improved by making a linear combination of symmetry-projected configurations constructed from a set of (generally non-orthogonal) deformed Slater determinants. Two extreme optimization strategies were considered: a FED approach where only the last-added determinant is optimized (along with the full set of linear variational coefficients), and a Res HF approach where all the variational parameters are optimized at once.

We note that our multi-component approach is exact in the limit of an infinite number of symmetry-projected configurations included in the expansion, regardless of the optimization strategy used. In such limit, the wavefunction coincides with the coherent state representation of the exact wavefunction

$$
|\Psi\rangle=\left(\int \prod_{p h} d z_{p h} d z_{p h}^{*} \mu(\mathbf{z})|\mathbf{z}\rangle\langle\mathbf{z}|\right)|\Psi\rangle,
$$

where $|\mathbf{z}\rangle$ is a generalized fermion coherent state $e^{45}$ generated from a Thouless rotation out of a reference Slater determinant $\left|\Phi_{0}\right\rangle$ (see, e.g., Ref. 22). Here, $\mu(\mathbf{z})$ is a measure guaranteeing that the closure relation (the term in parenthesis) equals the identity operator.

Our work has shown that for molecular systems a FED approach tends to be more efficient than a Res HF one in building ground state correlations, even if the latter yields a more elegant wavefunction. This is because convergence is easier and the optimization problem can be implemented with linear computational cost in the number of determinants. We have observed that a few symmetry-projected configurations are sufficient to account for most of the correlations (both weak and strong) in simple molecular systems, such as the nitrogen and the water molecule. Near equilibrium, we can even obtain variational energies (in small basis sets) that are near the broken symmetry coupled-cluster ones, deemed to be 
exact albeit with wavefunctions that do not possess the correct quantum numbers.

In addition, we have revisited the copper oxide cores as an example of a challenging multi-reference system where both static and dynamic correlations are significant. By using a FED expansion in terms of symmetry-projected configurations with good spatial and spin symmetries we were able to improve our results yielding a linear isomerization path with the same shape as those previously reported with RASPT2, CR-CC, or DMRG-SC-CTSD. The relative energy between the $\mu-\eta^{2}: \eta^{2}$-peroxodicopper(II) and the bis( $\mu$-oxo)-dicopper(II) forms is predicted to be somewhat larger than that predicted with RASPT2, CR-CC, or DMRG-SC-CTSD, at $\approx 50 \mathrm{kcal} / \mathrm{mol}$.

In the examples considered in this work, we have observed that spatial symmetry breaking and restoration bring in a very significant amount of correlation. If a molecule has high symmetry, static correlation from nearly degenerate configurations can be expected. This correlation can be largely accounted for by means of spatial symmetry restoration. It is only possible to carry out such spatial symmetry breaking and restoration in molecules with a symmetry group different from $C_{1}$ but evidently, this is consistent with the lack of point group static correlation in nonsymmetric cases. If one is interested in scanning over both symmetric and nonsymmetric regions of a potential energy surface for certain atomic configurations, we advocate using the point group of the least symmetric configuration. In this way, the energies thereby obtained would be continuous. Any method that exploits point group symmetry (e.g., complete active space selfconsistent field (CASSCF)) may suffer from discontinuities in changing from symmetric to non-symmetric configurations. Lowering the symmetry is one way to avoid these discontinuities although a certain amount of static correlation present in the high-symmetry configurations would remain unaccounted for. Furthermore, we have observed in previous work ${ }^{11,18}$ that breaking and restoring spatial symmetry in translationally invariant systems improves significantly the quality of the calculated multi-reference wavefunctions.

An interesting question that results from this work is to determine the most efficient prescription to account for these correlations. We have observed, for instance, that two S-UHF configurations tend to give energies that are of similar quality as a single S-GHF configuration, while the latter involves a computational effort that is roughly two orders of magnitude larger because of the size of the respective integration grids. This need not, however, be true for all systems: a frustrated configuration such as an equilateral $\mathrm{H}_{3}$ triangle will undoubtedly benefit significantly from the use of non-collinear deformed determinants. Given a determinantal expansion of a fixed length, letting all the determinants be independent will always afford the best description. Nevertheless, by constructing a same-size expansion in terms of the superposition of the Goldstone manifolds of fewer broken-symmetry states one may obtain a wavefunction that is near in quality to the former one. The latter has the virtue of respecting all symmetries of the Hamiltonian and being defined by a smaller number of computational parameters, thus becoming easier to optimize. Identifying those "efficient symme- tries" is certainly of paramount importance for practical applications.

\section{ACKNOWLEDGMENTS}

This work was supported by the Department of Energy, Office of Basic Energy Sciences, Grant No. DEFG02-09ER16053. G.E.S. is a Welch Foundation Chair (C0036). C.A.J.H. acknowledges support from the Lodieska Stockbridge Vaughn Fellowship.

\section{APPENDIX: MATRIX ELEMENTS BETWEEN SYMMETRY-PROJECTED CONFIGURATIONS}

In this appendix we provide explicit expressions for matrix elements between symmetry-projected configurations. We note that these can be expressed in terms of matrix elements between non-orthogonal Slater determinants, for which an extended Wick's theorem can be used as shown by, e.g., Blaizot and Ripka. ${ }^{22}$ A detailed derivation of the form of the matrix elements can be found in, e.g., Ref. 23.

We assume a non-relativistic, Born-Oppenheimer molecular electronic Hamiltonian $\hat{H}$ expressed in the form

$$
\hat{H}=\sum_{\mu \lambda} g_{\mu \lambda} a_{\mu}^{\dagger} a_{\lambda}+\frac{1}{4} \sum_{\mu \nu \lambda \sigma}\langle\mu \nu \| \lambda \sigma\rangle a_{\mu}^{\dagger} a_{\nu}^{\dagger} a_{\sigma} a_{\lambda},
$$

where $g_{\mu \lambda}$ are one-electron (core Hamiltonian) integrals and $\langle\mu \nu \| \lambda \sigma\rangle$ are anti-symmetrized two-electron integrals in Dirac notation. In addition, we use the matrix $C$ for molecular orbital coefficients. In the rest of this appendix we assume the use of an orthonormal basis; the transformation from the standard atomic orbital basis to an orthonormal one is straightforward.

Overlap and Hamiltonian matrix elements between symmetry-projected configurations of the form $\hat{P}_{k k^{\prime}}^{j}|\Phi\rangle$ are expressed in terms of norm and Hamiltonian overlaps between rotated determinants $\hat{R}(\vartheta)|\Phi\rangle$ as

$$
\begin{array}{r}
\left\langle{ }^{r} \Phi \mid \hat{P}_{k k^{\prime}}^{j}{ }^{s} \Phi\right\rangle=\frac{1}{V} \int_{V} d \vartheta w_{k k^{\prime}}^{j}(\vartheta) n^{r s}(\vartheta), \\
\left\langle{ }^{r} \Phi\left|\hat{H} \hat{P}_{k k^{\prime}}^{j}\right|^{s} \Phi\right\rangle=\frac{1}{V} \int_{V} d \vartheta w_{k k^{\prime}}^{j}(\vartheta) n^{r s}(\vartheta) h^{r s}(\vartheta),
\end{array}
$$

where the left superscript $r$ in $\left|{ }^{r} \Phi\right\rangle$ is used as to label the broken symmetry determinant. Here,

$$
\begin{aligned}
& n^{r s}(\vartheta) \equiv\left\langle{ }^{r} \Phi|\hat{R}(\vartheta)|^{s} \Phi\right\rangle, \\
& h^{r s}(\vartheta) \equiv \frac{\left\langle{ }^{r} \Phi|\hat{H} \hat{R}(\vartheta)|^{s} \Phi\right\rangle}{\left\langle{ }^{r} \Phi|\hat{R}(\vartheta)|^{s} \Phi\right\rangle} .
\end{aligned}
$$

The norm overlaps of Eq. (A3a) can be evaluated with

$$
\begin{aligned}
& n^{r s}(\vartheta)=\operatorname{det} X^{r s}(\vartheta), \\
& X^{r s}(\vartheta)=C^{r \dagger} R(\vartheta) C^{s} .
\end{aligned}
$$

Here, $C^{r}$ is the set of occupied molecular orbital coefficients in $\left.\left.\right|^{r} \Phi\right\rangle$ and is therefore a rectangular matrix of dimension 
$M \times N$. In addition, $R(\vartheta)$ is the matrix representation of the rotation operator $\hat{R}(\vartheta)$ in the single-particle basis (dimension $M \times M)$. The resulting $X^{r s}(\vartheta)$ is thus an $N \times N$ matrix.

The Hamiltonian overlaps of Eq. (A3b) are given by

$$
\begin{aligned}
& h^{r s}(\vartheta)=\sum_{\mu \lambda}\left[g_{\mu \lambda}+\frac{1}{2} G_{\mu \lambda}^{r s}(\vartheta)\right] \rho_{\lambda \mu}^{r s}(\vartheta), \\
& G_{\mu \lambda}^{r s}(\vartheta)=\sum_{\nu \sigma}\langle\mu \nu \| \lambda \sigma\rangle \rho_{\sigma \nu}^{r s}(\vartheta),
\end{aligned}
$$

where we have expressed them in terms of the transition density matrix $\rho^{r s}(\vartheta)$. The latter can be built according to

$$
\rho^{r s}(\vartheta)=R(\vartheta) C^{s}\left[X^{r s}(\vartheta)\right]^{-1} C^{r \dagger} .
$$

Here, as before, the matrix $C^{r}$ is the rectangular matrix of occupied molecular orbital coefficients in $\left|{ }^{r} \Phi\right\rangle$.

Matrix elements appearing in contributions to the energy gradient can also be expressed in terms of overlaps between rotated determinants:

$$
\begin{aligned}
& \left\langle{ }^{r} \Phi_{h}^{p}\left|\hat{P}_{k k^{\prime}}^{j}\right| s\right|=\frac{1}{V} \int_{V} d \vartheta w_{k k^{\prime}}^{j}(\vartheta) n^{r s}(\vartheta) N_{p h}^{r s}(\vartheta), \\
& \left\langle{ }^{r} \Phi_{h}^{p}\left|\hat{H} \hat{P}_{k k^{\prime}}^{j}\right|{ }^{s} \Phi\right\rangle=\frac{1}{V} \int_{V} d \vartheta w_{k k^{\prime}}^{j}(\vartheta) n^{r s}(\vartheta) H_{p h}^{r s}(\vartheta),
\end{aligned}
$$

where $\left|{ }^{r} \Phi_{h}^{p}\right\rangle$ represents a one-particle excitation $(h \rightarrow p)$ of the determinant $\left|{ }^{r} \Phi\right\rangle$. Here,

$$
\begin{aligned}
& N_{p h}^{r s}(\vartheta) \equiv \frac{\left\langle{ }^{r} \Phi_{h}^{p}|\hat{R}(\vartheta)|^{s} \Phi\right\rangle}{\left\langle{ }^{r} \Phi|\hat{R}(\vartheta)|^{s} \Phi\right\rangle}, \\
& H_{p h}^{r s}(\vartheta) \equiv \frac{\left\langle{ }^{r} \Phi_{h}^{p}|\hat{H} \hat{R}(\vartheta)|^{s} \Phi\right\rangle}{\left\langle{ }^{r} \Phi|\hat{R}(\vartheta)|^{s} \Phi\right\rangle} .
\end{aligned}
$$

The matrix elements of Eq. (A10) are given by

$$
\begin{aligned}
N_{p h}^{r s}(\vartheta)= & {\left[C^{r \dagger} \rho^{r s}(\vartheta) C^{r}\right]_{p h}, } \\
H_{p h}^{r s}(\vartheta)= & h^{r s}(\vartheta)\left[C^{r \dagger} \rho^{r s}(\vartheta) C^{r}\right]_{p h} \\
& +\left[C^{r \dagger}\left(\mathbf{1}-\rho^{r s}(\vartheta)\right) f^{r s}(\vartheta) \rho^{r s}(\vartheta) C^{r}\right]_{p h},
\end{aligned}
$$

(A11b)

where we have set $f_{\mu \lambda}^{r s}(\vartheta)=g_{\mu \lambda}+G_{\mu \lambda}^{r s}(\vartheta)$.

${ }^{1}$ C. A. Jiménez-Hoyos, T. M. Henderson, T. Tsuchimochi, and G. E. Scuseria, J. Chem. Phys. 136, 164109 (2012).

${ }^{2}$ K. Samanta, C. A. Jiménez-Hoyos, and G. E. Scuseria, J. Chem. Theory Comput. 8, 4944 (2012).

${ }^{3}$ A. J. Garza, C. A. Jiménez-Hoyos, and G. E. Scuseria, J. Chem. Phys. 138, 134102 (2013).

${ }^{4}$ P. Rivero, C. A. Jiménez-Hoyos, and G. E. Scuseria, J. Phys. Chem. B 117, 12750 (2013).

${ }^{5}$ P. Rivero, C. A. Jiménez-Hoyos, and G. E. Scuseria, J. Phys. Chem. A 117, 8073 (2013).

${ }^{6}$ K. W. Schmid, R.-R. Zheng, F. Grümmer, and A. Faessler, Nucl. Phys. A 499, 63 (1989).
${ }^{7}$ K. W. Schmid, Prog. Part. Nucl. Phys. 52, 565 (2004).

${ }^{8}$ H. Fukutome, Prog. Theor. Phys. 80, 417 (1988).

${ }^{9}$ H. Koch and E. Dalgaard, Chem. Phys. Lett. 212, 193 (1993).

${ }^{10}$ N. Tomita, S. Ten-no, and Y. Tanimura, Chem. Phys. Lett. 263, 687 (1996).

${ }^{11}$ R. Rodríguez-Guzmán, C. A. Jiménez-Hoyos, R. Schutski, and G. E. Scuseria, Phys. Rev. B 87, 235129 (2013).

${ }^{12}$ N. Tomita and S. Watanabe, Phys. Rev. Lett. 103, 116401 (2009).

${ }^{13}$ N. Tomita, Phys. Rev. B 69, 045110 (2004).

${ }^{14}$ A. Ikawa, S. Yamamoto, and H. Fukutome, J. Phys. Soc. Jpn. 62, 1653 (1993).

${ }^{15}$ R. Broer and W. C. Nieuwpoort, Theor. Chim. Acta 73, 405 (1988).

${ }^{16}$ E. Hollauer and M. A. C. Nascimento, J. Chem. Phys. 99, 1207 (1993).

${ }^{17}$ P. Ring and P. Schuck, The Nuclear Many-Body Problem (Springer-Verlag, Berlin, 1980).

${ }^{18}$ R. Rodríguez-Guzmán, K. W. Schmid, C. A. Jiménez-Hoyos, and G. E. Scuseria, Phys. Rev. B 85, 245130 (2012).

${ }^{19}$ K. W. Schmid, F. Grümmer, and A. Faessler, Phys. Rev. C 29, 291 (1984).

${ }^{20}$ P.-O. Löwdin, in Quantum Theory of Atoms, Molecules, and the Solid State: A Tribute to John C. Slater, edited by P.-O. Löwdin (Academic Press, New York, 1966), pp. 601-623.

${ }^{21}$ I. Mayer, Adv. Quantum Chem. 12, 189 (1980).

${ }^{22}$ J.-P. Blaizot and G. Ripka, Quantum Theory of Finite Systems (The MIT Press, Cambridge, MA, 1985).

${ }^{23}$ C. A. Jiménez-Hoyos, "Variational approaches to the molecular electronic structure problem based on symmetry-projected Hartree-Fock configurations," Ph.D. thesis (Rice University, 2013).

${ }^{24}$ C. A. Jiménez-Hoyos, R. Rodríguez-Guzmán, and G. E. Scuseria, "Excited electronic states from a variational approach based on symmetryprojected Hartree-Fock configurations," J. Chem. Phys. (submitted); preprint arXiv:1309.6343 (2013).

${ }^{25}$ A. Szabo and N. S. Ostlund, Modern Quantum Chemistry: Introduction to Advanced Electronic Structure Theory (Dover Publications, Mineola, NY, 1996).

${ }^{26}$ H. J. Mang, Phys. Rep. 18, 325 (1975).

${ }^{27}$ C. A. Jiménez-Hoyos, R. Rodríguez-Guzmán, and G. E. Scuseria, Phys. Rev. A 86, 052102 (2012).

${ }^{28}$ S. Ten-no, Theor. Chem. Acc. 98, 182 (1998).

${ }^{29}$ S. Yamamoto, A. Takahashi, and H. Fukutome, J. Phys. Soc. Jpn. 60, 3433 (1991).

${ }^{30}$ M. J. Frisch, G. W. Trucks, H. B. Schlegel, et al., Gaussian 09, Gaussian Development Version, Revision H.1, Gaussian Inc., Wallingford, CT, 2009.

${ }^{31}$ J. L. Egido, J. Lessing, V. Martin, and L. M. Robledo, Nucl. Phys. A 594, 70 (1995).

${ }^{32}$ D. C. Liu and J. Nocedal, Math. Program. 45, 503 (1989).

${ }^{33}$ J. Nocedal, Math. Comput. 35, 773 (1980).

${ }^{34}$ K. P. Huber, and G. Herzberg, in NIST Chemistry Webbook, NIST Standard Reference Database Vol. 69, edited by P. J. Linstrom and W. G. Mallard (National Institute of Standards and Technology, Gaithersburg, MD, 2011), see http://webbook.nist.gov/ (retrieved April 1, 2013).

${ }^{35}$ H. Larsen, J. Olsen, P. Jørgensen, and O. Christiansen, J. Chem. Phys. 113, 6677 (2000)

${ }^{36} \mathrm{Near} r_{\mathrm{eq}}$, CCSD yields a 12.3 mhartree energy difference once both effects are accounted for.

${ }^{37}$ J. Linderberg and Y. Öhrn, Int. J. Quantum Chem. 12, 161 (1977).

${ }^{38}$ J. Olsen, P. Jørgensen, H. Koch, A. Balkova, and R. J. Bartlett, J. Chem. Phys. 104, 8007 (1996).

${ }^{39}$ At $r_{\text {eq }}$, CCSD is 3.5 mhartree lower in the Cartesian basis set.

${ }^{40}$ C. J. Cramer, M. Włoch, P. Piecuch, C. Puzzarini, and L. Gagliardi, J. Phys. Chem. A 110, 1991 (2006).

${ }^{41}$ P. ̊. Malmqvist, K. Pierloot, A. R. M. Shahi, C. J. Cramer, and L. Gagliardi, J. Chem. Phys. 128, 204109 (2008).

${ }^{42}$ T. Yanai, Y. Kurashige, E. Neuscamman, and G. K.-L. Chan, J. Chem. Phys. 132, 024105 (2010).

${ }^{43}$ D. G. Liakos and F. Neese, J. Chem. Theory Comput. 7, 1511 (2011).

${ }^{44}$ The use of Cartesian basis functions lowers the UHF energy at $f=100$ by 3.2 mhartree.

${ }^{45}$ J.-P. Gazeau, Coherent States in Quantum Physics (Wiley-VCH, Weinheim, 2009). 